\title{
Atomic force microscope featuring an integrated optical microscope
}

\author{
Constant A.J. Putman *, Kees O. van der Werf, Bart G. de Grooth, Niek F. van Hulst, \\ Frans B. Segerink and Jan Greve \\ Department of Applied Physics, University of Twente, P.O. Box 217, 7500 AE Enschede, The Netherlands
}

Received 12 August 1991

\begin{abstract}
The atomic force microscope (AFM) is used to image the surface of both conductors and nonconductors. Biological specimens constitute a large group of nonconductors. A disadvantage of most AFM's is the fact that relatively large areas of the sample surface have to be scanned to pinpoint a biological specimen (e.g. cell, chromosome) of interest. The AFM presented here features an incorporated optical microscope. Using an $X Y$-stage to move the sample, an object is selected with the aid of the optical microscope and a high-resolution image of the object can be obtained using the AFM. Results on chromosomes and cells demonstrate the potential of this instrument. The microscope further enables a direct comparison between optically observed features and topological information obtained from AFM images.
\end{abstract}

\section{Introduction}

Since the introduction of the atomic force microscope (AFM) [1] a large number of biological objects with dimensions ranging from DNA molecules [2] to blood cells [3] have been imaged utilizing the high resolution of this technique. A major advantage of the AFM over other techniques with similar resolution such as scanning tunneling microscopy (STM) and scanning electron microscopy (SEM) is that the AFM requires no coating of the specimen. Furthermore, Hansma and co-workers demonstrated that the AFM can be operated in an aqueous environment [4]. This holds great promise for biological studies under physiological conditions. In practice, most operating time of the AFM is used to locate specific objects of interest, which are more or less randomly distributed on the sample substrate, by making multiple AFM images. For objects with a size of $1 \mu \mathrm{m}$ or larger, this problem has been solved by building an AFM which has an inte-

\footnotetext{
* To whom correspondence should be directed.
}

grated inverted optical microscope (EPI-illumination). With this instrument an object of interest can be selected quickly using the optical microscope and a high-resolution AFM image can be obtained of the selected object. A direct comparison between the optical image (in reflection or in fluorescence) and the AFM image can be made due to the overlapping field of view.

\section{Experimental set-up and first results}

A problem encountered in integrating an optical microscope in an AFM is that there is not much space near the sample-tip location to position an objective lens. In one commercially available AFM [5] this has been solved by using special long-working-distance objectives. A disadvantage of this solution is the fact that the tip and cantilever are directly located on top of the object thus obscuring the optical image of the object. This problem can be circumvented by placing a small objective lens inside the hollow piezo tube. Since most biological objects are transparent, an 


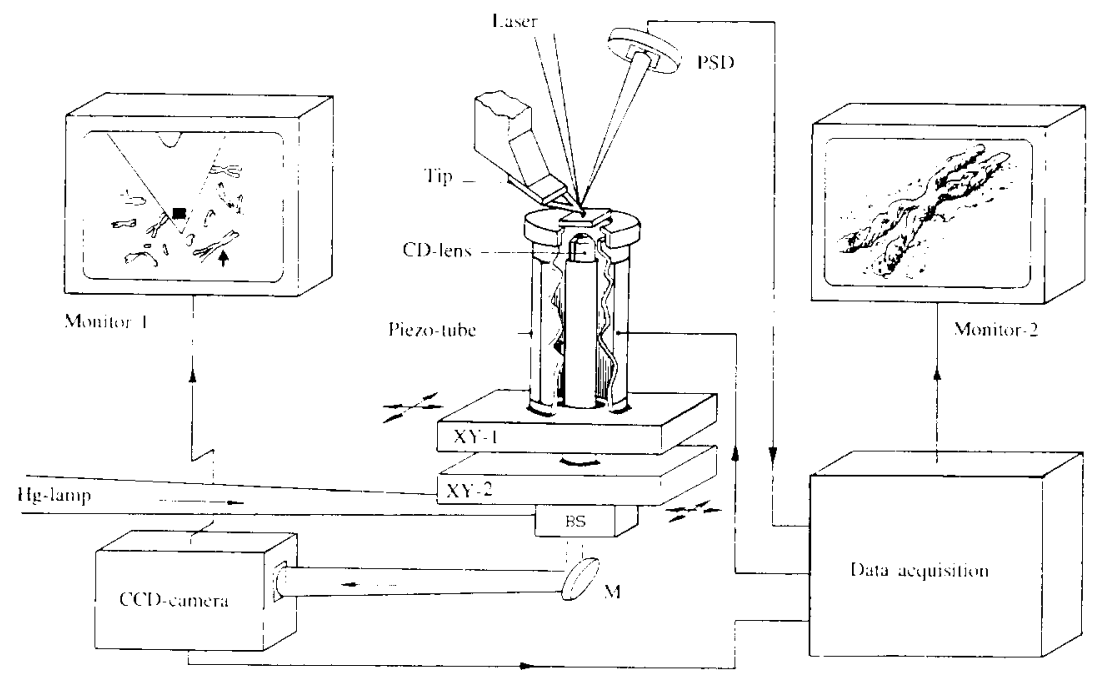

Fig. 1. Schematic representation of the AFM integrated with an optical microscope. The optical microscope consists of a $\mathrm{m}$. arc lamp for illumination, a beam splitter BS, a beam steering mirror $(\mathrm{M})$, an objective lens (numerical aperture = 1.4), CCD-camera with video monitor, Monitor- 1 . The lens can be moved by an $X Y$-stage (XY-2, range: $2 \times 2 \mathrm{~mm}^{2}$ ). The CD-lens can also be translated in the $Z$-direction in order to focus the sample. The AFM consists of a piezo tube for sample scanning and a cantilever-tip. The displacement of the cantilever is measured by the optical beam deflection method [11] using a diode laser and a quadrant detector, PSD. The AFM images are displayed on Monitor-2. The sample can be moved over a range of $3 \times 3 \mathrm{~mm}{ }^{2}$ by a second $X Y$-stage (XY-1).

optical image can be obtained from the back side by using a transparent object carrier, e.g. a microscope slide. In fig. 1 a schematic representation of the set-up is shown. The instrument is described in detail elsewhere [6]. An object field with a size of $3 \times 3 \mathrm{~mm}^{2}$ can be screened by

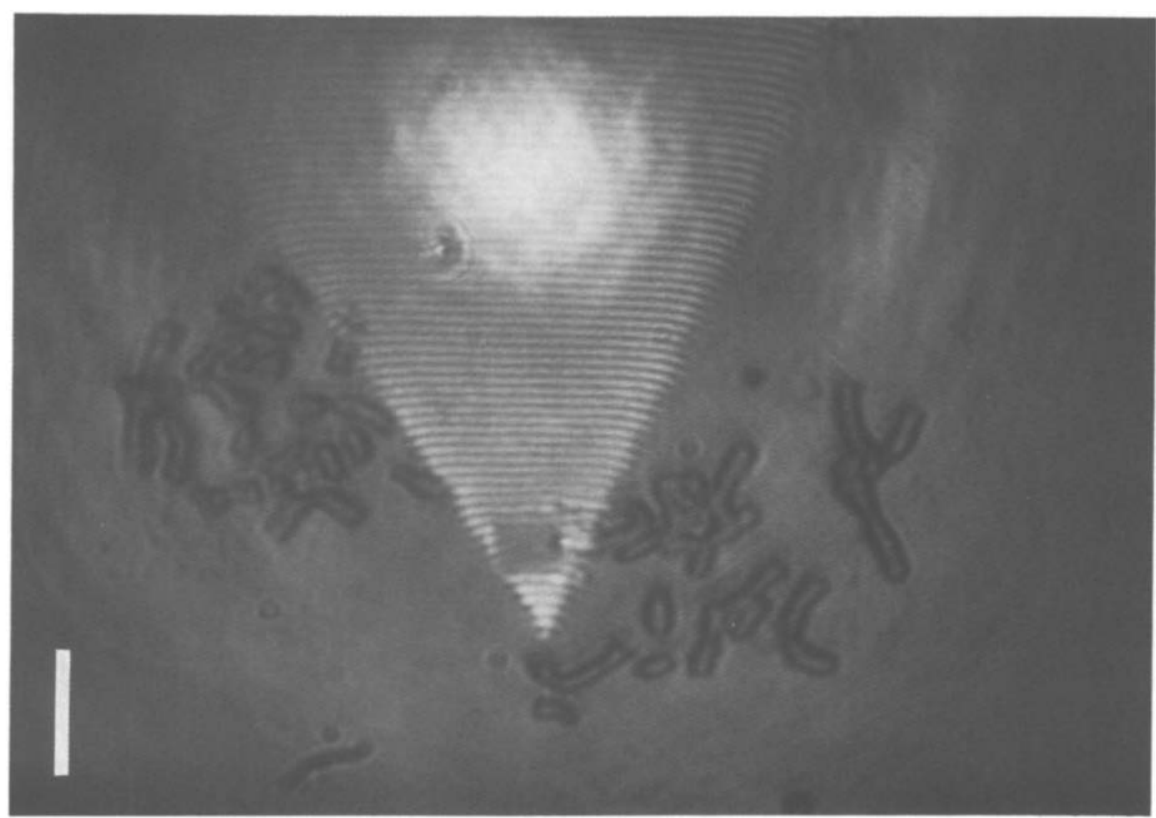

Fig. 2. Optical image in reflection of a sample of metaphase chromosomes taken from a Chinese hamster lung cell line (CHL-V79), prepared according to the method described elsewhere [12]. Bar: $10 \mu \mathrm{m}$. 
moving the sample with an $X Y$-translation stage. The field of view of the optical microscope is 100 $\mu \mathrm{m}$ in diameter with an optical resolution of about $1 \mu \mathrm{m}$. The maximum scan range of the AFM is $15 \times 15 \mu \mathrm{m}^{2}$ and the resolution is a few nanometers (no extensive noise-reducing precautions have been taken yet).

An optical image (in reflection) of a sample of metaphase chromosomes is shown in fig. 2. The $\mathrm{V}$-shaped micro-fabricated cantilever $\left(\mathrm{Si}_{3} \mathrm{~N}_{4}\right)$ [7] is clearly observed. The $4 \times 4 \mu \mathrm{m}^{2}$ square at the apex of the cantilever is the actual pyramidal tip with a radius of typically $30 \mathrm{~nm}$ [5]. The interference pattern (Newton fringes) within the boundaries of the cantilever originate from the interference between light reflected from the sample carrier surface and the tilted cantilever. The observed spacing of the fringes can be used to calculate the tilt angle of the cantilever. With the relatively monochromatic light used for illumination (Mercury arc lamp, $546 \mathrm{~nm}$ ) the angle is about $15^{\circ}$ and in agreement with the angle installed in the instrument. The white spot is from the laser beam sensing the cantilever displacement.
When a biological object of interest is located using the optical microscope, the object is positioned under the AFM tip and an AFM image is obtained. In fig. 3 an AFM image of a metaphase chromosome that was selected from the optical image is shown. We see that the chromatids of the chromosome display a pattern of loops with a period of $800 \mathrm{~nm}$. This is in agreement with earlier observations on coiling of the chromatids [8,9]. Higher-resolution AFM images reveal structures with sizes in the range of 50 to $60 \mathrm{~nm}[6]$. This example clearly shows the possibilities of the instrument.

As a second example an AFM image of a white blood cell is shown (fig. 4). A blood droplet is smeared on a microscope slide and air-dried. Amongst a multitude of red blood cells (ratio 4000 to 1) a lymphocyte is selected using the optical microscope. The large nucleus is clearly visible.

Other preliminary results include images of a neutrophilic granulocyte. After air-drying for a day the lobed nucleus is observed in the topology of the AFM image. No high resolution of the nucleus is obtained because the cell membrane

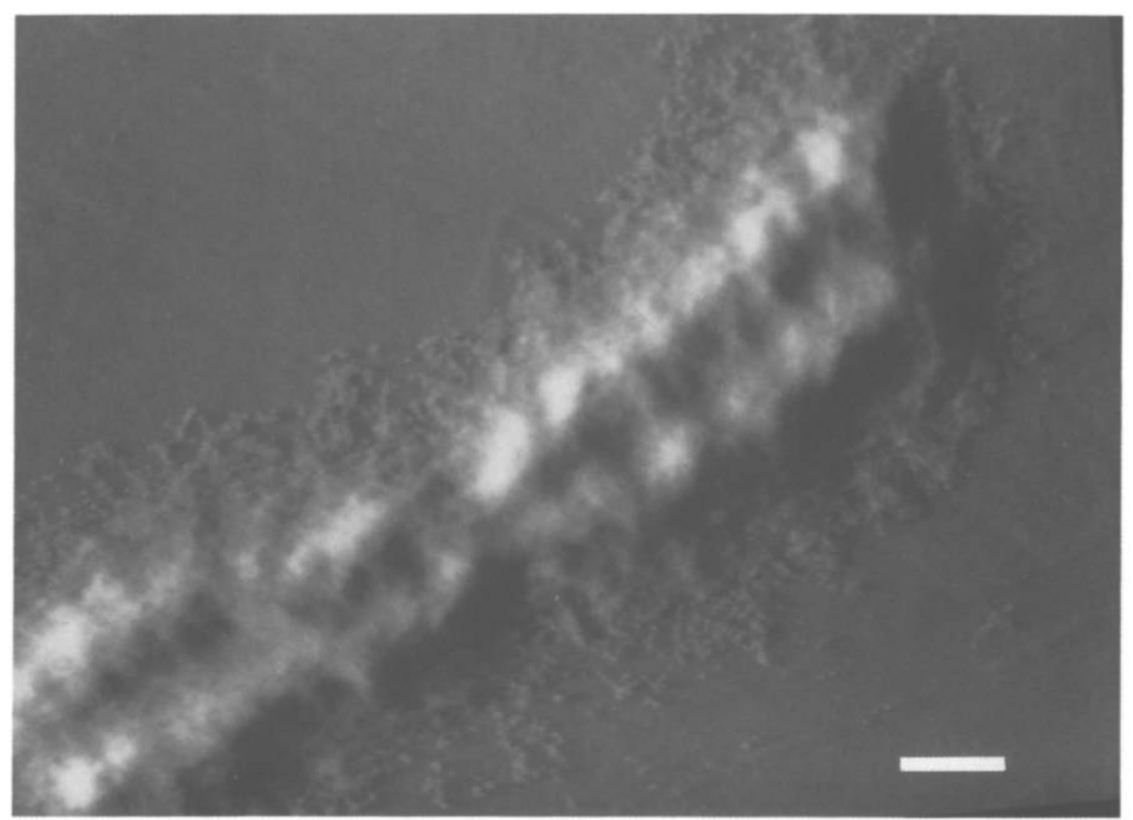

Fig. 3. AFM image of a single CHL metaphase chromosome. The applied force is $5 \mathrm{nN}$. Bar: $1 \mu \mathrm{m}$. 


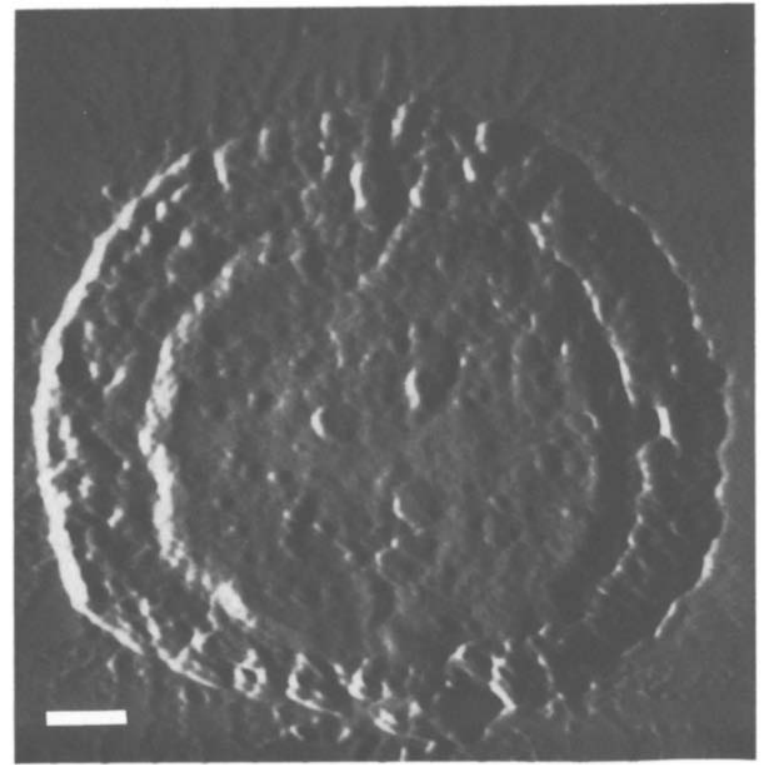

Fig. 4. AFM image of a lymphocyte. The applied force is 10 $\mathrm{nN}$. Bar: $1 \mu \mathrm{m}$.

covers it. On treated chromosomes banding patterns can be observed using an optical microscope. The first results obtained by the AFM show that this is expressed in height variations. Thus we are able to correlate the optically observed banding patterns and the topology as observed by the AFM of treated chromosomes.

We are currently investigating the chromosome appearance under different physiological conditions, such as the influence of divalent cations [10], in order to obtain a better understanding of the chromosome structure.

\section{Conclusions}

The atomic force microscope with integrated optical microscope as presented, is an instrument that is easy to operate and can be a useful research tool for biologists to study biological struc- tures and processes on a molecular scale under physiological conditions. The instrument reduces the operating time significantly. It further enables direct comparison between optically observed features and the topology.

\section{Acknowledgements}

We thank G.M.J. Segers and Y.M. Kraan for skillful sample preparation and G. van Oort, G.J. Puppels and $M$. van Graft for stimulating discussions. This research was supported by the Netherlands Organization for Scientific Research (NWO).

\section{References}

[1] G. Binnig, C.F. Quate and Ch. Gerber, Phys. Rev. Lett. 56 (1986) 930.

[2] S.M. Lindsay, L.A. Nagahara, T. Thundat, U. Knipping, R.L. Rill, B. Drake, C.B. Prater, A.L. Weisenhorn, S.A.C. Gould and P.K. Hansma, Biom. Struct. Dyn. 7 (1989) 279.

[3] S.A.C. Gould, B. Drake, C.B. Prater, A.L. Weisenhorn, S. Manne, H.G. Hansma, P.K. Hansma, J. Massie, M. Longmire, V. Elings, B. Dixon Northern, B. Mukergee, C.M. Peterson, W. Stoeckenius, T.R. Albrecht and C.F. Quate, J. Vac. Sci. Technol. A 8 (1990) 369.

[4] B. Drake, C.B. Prater, A.L. Weisenhorn, S.A.C. Gould, T.R. Albrecht, C.F. Quate, D.S. Cannell, H.G. Hansma and P.K. Hansma, Science 243 (1989) 1586.

[5] Park Scientific Instruments, Mountain View, CA, USA.

[6] C.A.J. Putman, K.O. van der Werf, B.G. de Grooth, N.F. van Hulst, F.B. Segerink and J. Greve, Rev. Sci. Instr., in press.

[7] T.R. Albrecht, S. Akamine, T.E. Carver and C.F. Quate, J. Vac. Sci. Technol. A 8 (1990) 3386.

[8] E. Boy de la Tour and U.K. Laemmli, Cell 55 (1988) 937.

[9] A.L. Bak, J. Zeuthen and F.H.C. Crick, Proc. Natl. Acad. Sci. USA 74 (1977) 1595.

[10] K.W. Adolph and L.R. Kreisman, Exp. Cell Res. 147 (1983) 155.

[11] G. Meyer and N.M. Amer, Appl. Phys. Lett. 53 (1988) 2400.

[12] J.C.K. Lee and G.F. Bahr, Chromosoma 88 (1983) 374. 